\title{
Maximum velocity of self-propulsion for an active segment
}

\author{
P. Recho a,b ${ }^{\mathrm{a}}$ L. Truskinovsky ${ }^{\mathrm{a}}$ \\ ${ }^{a}$ LMS, CNRS-UMR 7649, Ecole Polytechnique, Route de Saclay, 91128 Palaiseau, France \\ ${ }^{b}$ Physicochimie Curie, CNRS-UMR168, Institut Curie, Centre de Recherche, 26 rue d'Ulm F-75248 Paris Cedex 05, France
}

\begin{abstract}
The motor part of a crawling eukaryotic cell can be represented schematically as an active continuum layer. The main active processes in this layer are protrusion, originating from non-equilibrium polymerization of actin fibers, contraction, induced by myosin molecular motors and attachment due to active bonding of trans-membrane proteins to a substrate. All three active mechanisms are regulated by complex signaling pathways involving chemical and mechanical feedback loops whose microscopic functioning is still poorly understood. In this situation, it is instructive to take a reverse engineering approach and study a problem of finding the spatial organization of standard active elements inside a crawling layer ensuring an optimal cost-performance trade-off. In this paper we assume that (in the range of interest) the energetic cost of self-propulsion is velocity independent and adopt, as an optimality criterion, the maximization of the overall velocity. We then choose a prototypical setting, formulate the corresponding variational problem and obtain a set of bounds suggesting that radically different spatial distributions of adhesive complexes would be optimal depending on the domineering active mechanism of self-propulsion. Thus, for contraction-dominated motility, adhesion has to cooperate with 'pullers' which localize at the trailing edge of the cell, while for protrusion-dominated motility it must conspire with 'pushers' concentrating at the leading edge of the cell. Both types of crawling mechanisms were observed experimentally.
\end{abstract}

\section{Introduction}

Eukariotic cells are spatially extended active bodies that can steadily self-propel in viscous environments at low Reynolds numbers [1, 2]. It has been understood $[3,4,5]$ that in these conditions a combination of stationarity and linearity of friction leads to kinematic reversibility and that a symmetric (under time reversal) stroke cannot produce self-propulsion. For Stokes swimmers, a variety of non-symmetric motility strategies have been proposed and optimized using various efficiency criteria $[6,7,8,9,10,11,12]$; similar models for crawlers advancing on a frictional background were considered in $[13,14,15]$. Most of the self-propulsion mechanisms proposed in these papers are fully kinematic in the sense that the time dependence of the shape of a swimmer/crawler is prescribed. This implies that appropriately chosen actuators can always perform the required internal movements. In cells the role of such actuators is played by active agents and in this paper we focus on the fact that their dynamics, while being driven biochemically, must be compatible with the fundamental balances of mass and momentum.

In the context of eukaryotic cell motility, a prototypical scheme of self-propulsion includes protrusion through polymerization of actin filaments which is accompanied by dynamic assembly of focal adhesions; myosin-driven contraction of the actin network which allows the motor part to advance a cargo, and, finally, detachment of adhesive contacts with a simultaneous depolymerization of actin fibers [16]. It is usually assumed that active polymerization ensuring protrusion can be described as the work of spatially distributed pushers, generating positive force couples, while active contraction can be viewed as an outcome of the mechanical action of distributed pullers, responsible for negative force couples [17, 18, 19, 20,21]. The role of ATP in reversible adhesion of adhesive patches (focal adhesions) is understood rather poorly and they are usually treated as passive viscous binders whose spatial distribution may be regulated actively [22] .

Email address: trusk@lms.polytechnique.fr (L. Truskinovsky) 
Since our knowledge of the mechanism controlling the transport and the intensity of active agents performing protrusion, contraction and adhesion is rather limited, we adopt in this paper a semi-kinematic approach and treat the corresponding distributions as functional control parameters constrained by the fundamental mechanical balances. We then pose a variational problem of finding the optimal temporal and spatial distributions of these parameters inside a crawling continuum body. In view of some successful attempts to justify such reverse engineering approach [23], we anticipate that our optimal solutions will be eventually backed by an appropriate constitutive theory.

While the real organisms are expected to optimize some measure of a trade-off between the velocity of selfpropulsion and the corresponding energy expenditure, in this paper we make a simplifying assumption that the energetic cost of self-propulsion is fixed and use as optimality criterion the maximization of the overall velocity. We are interested in steady translocation and assume that the internal distributions of mechanical parameters are compatible with the traveling wave ansatz. This simplifying assumption allows us to replace the optimization of the crawling stroke in space and time by a purely spatial optimization of the internal distribution of active elements in the co-moving coordinate system. In the interest of analytic transparency we use the simplest 1D model of actively contracting continuum (subjected to viscous frictional forces) that has been used repeatedly in the cell motility studies $[24,25,26,27,28,29,30,16,31]$. In a setting similar to ours the dependence of cell velocity on the distribution of active stresses and adhesion properties was studied in [18] where both contraction and protrusion were represented by active couples. Given that protrusion is usually localized at the leading edge of the cell, we model the effect of active polymerization differently by using Stefan type boundary conditions on the edges of a crawling segment that fix the influx and the outflow of actin, see also [32, 33, 21]. For the given strength of protrusion, we prescribe the average level of contractile activity, and then search for the optimal internal distribution of contractile and adhesive units.

Our analysis of the ensuing variational problem demonstrates that radically different distributions of focal adhesions are optimal depending on the domineering active mechanism of self-propulsion. Thus, for contractiondominated motility, focal adhesions have to cooperate with pullers which end up localizing at the trailing edge of the cell while for protrusion-dominated motility they must conspire with pushers which concentrate in our model at the leading edge of the cell. Both types of crawling mechanisms have been observed experimentally.

The paper is organized as follows. In Section 2 we formulate the model. A simple analytically tractable case of contraction dominated motility is treated in Section 3. In Section 4 we obtain analytically upper and low bounds for the self-propulsion velocity in the general case. The same optimization problem was studied numerically in Section 5. In Section 6 we present some evidence that our lower bound may in fact coincide with the optimal solution. The last Section 7 contains the discussion of our results.

\section{The model}

Following [29, 32], we consider a one-dimensional segment of viscous active gel representing the cell lamellipodium on a frictional substrate. The segment has two free boundaries which we identify as the trailing edge $l_{-}(t)$ and the leading edge $l_{+}(t)$. The force balance can be written in the form

$$
\partial_{x} \sigma=\xi v
$$

where $\sigma(x, t)$ is the stress and $v(x, t)$ is the velocity. We assume that the frictional coefficient mimicking the distribution of focal adhesions $[33,34,32,35,36,37]$ is space and time dependent $\xi(x, t) \geq 0$. The constitutive behavior of the gel is modeled by the equation [29, 32]

$$
\sigma=\eta \partial_{x} v+\chi
$$

where the active pre-stress $\chi(x, t) \geq 0$ accounting for the presence of myosin molecular motors [38, 39, 40, 41, 42] is also assumed to be a function of space and time. For simplicity we assume that the bulk viscosity coefficient $\eta>0$ is constant. The assumption of infinite compressibility in (2) allows us to decouple the transport of (actin) density $\rho(x, t)$ from force balance making the mechanical problem 'statically determinate'. The mass balance equation $\partial_{t} \rho+\partial_{x}(\rho v)=0$ can then be solved independently after the velocity field is determined [43, 44].

We further assume that some internal mechanism (stiffness of the cell cortex [45, 46, 47, 48, 49, 50], osmotic pressure actively controlled by the channels and pumps on the cell membrane [51, 52], etc.) maintains a given size $L_{0}=l_{+}-l_{-}$of the cell. Therefore the stress at the edges must be the same $\sigma\left(l_{-}(t), t\right)=\sigma\left(l_{+}(t), t\right)=\sigma_{0}$, where $\sigma_{0}(t)$ is then an unknown function. To model active protrusion we impose two kinematic Stefan type boundary conditions 
characterizing the rate of actin polymerization $v_{+}>0$ and depolymerization $v_{-}>0$ on the boundaries of the moving segment $[29,34,32,33,43]$

$$
i_{ \pm}=v_{ \pm}+v\left(l_{ \pm}(t), t\right) \text {. }
$$

For consistency, the overall mass balance must be also respected on the moving boundaries and we set $\rho\left(l_{-}(t), t\right) v_{-}=$ $\rho\left(l_{+}(t), t\right) v_{+}$, which implies an instantaneous recycling of depolymerized actin from the trailing edge to the leading edge, see [21, 43] for more detail on such closure of the treadmilling cycle. While there is considerable experimental evidence that active polymerization is indeed localized at the leading edge of a crawling cell, the de-polymerization may be spread along the length of the lamellipodium $[33,32]$. However, in the interest of analytic transparency, such spreading will be ignored in this study, see though [43].

The two functions $\chi$ and $\xi$ can be interpreted as infinite dimensional controls parameters and found through an optimization procedure. Even in the absence of a detailed microscopic model governing the rearrangement of these agents we still need to subject them to integral constraints prescribing the average number of adhesion complexes [42]

$$
\frac{1}{L_{0}} \int_{l_{-}(t)}^{l_{+}(t)} \xi(x, t) d x=\xi^{*},
$$

where $\xi^{*}>0$ is a given constant and

$$
\frac{1}{L_{0}} \int_{l_{-}(t)}^{l_{+}(t)} \chi(x, t) d x=\chi^{*},
$$

where $\chi^{*}>0$ is another given constant representing the average number of contractile motors [53] . It is clear from $(4,5)$ that since we prescribe the density of active agents, the performance of the self-propulsion machinery will be proportional to the length of the active segment, so the appropriate velocity functional may be also normalized by the total length.

To simplify the analysis we further assume that the motion of the active segment is steady [32, 33] with unknown velocity $V=\dot{l}_{-}=\dot{l}_{+}$and that the unknown functions $\sigma, v$ and the unknown controls $\xi, \chi$ depend exclusively on the appropriately chosen co-moving coordinate $u=\left(x-x_{0}-V t\right) / L_{0} \in[-1 / 2,1 / 2]$. Then in dimensionless variables $\sigma:=\sigma / \chi^{*}, x:=x / \sqrt{\eta / \xi^{*}}, t:=t /\left(\eta / \chi^{*}\right), \xi:=\xi / \xi^{*}$ and $\chi:=\chi / \chi^{*}$ we obtain the force balance equation

$$
-\frac{1}{L^{2}} \partial_{u}\left(\frac{\partial_{u} \sigma(u)}{g_{1}(u)}\right)+\sigma(u)=g_{2}(u),
$$

where $L:=L_{0} / \sqrt{\eta / \xi^{*}}$. The re-scaled control functions

$$
g_{1}(u)=\xi(L u) \geq 0, g_{2}(u)=\chi(L u) \geq 0
$$

must satisfy the constraints

$$
\int_{-1 / 2}^{1 / 2} g_{1}(u) d u=\int_{-1 / 2}^{1 / 2} g_{2}(u) d u=1 .
$$

The boundary conditions take the form

$$
\left\{\begin{array}{c}
\sigma(-1 / 2)=\sigma(1 / 2) \\
\frac{1}{L^{2}}\left(\frac{\partial_{u} \sigma(1 / 2)}{g_{1}(1 / 2)}-\frac{\partial_{u} \sigma(-1 / 2)}{g_{1}(-1 / 2)}\right)=-\overline{\Delta V}
\end{array}\right.
$$

where

$$
\overline{\Delta V}:=\frac{\left(v_{+}-v_{-}\right) \eta}{\chi^{*} L_{0}} .
$$

The dimensionless velocity of the segment per length $\bar{V}=V / L$ can be found from the formula

$$
\bar{V}=\overline{V_{m}}+\frac{1}{2 L^{2}}\left(\frac{\partial_{u} \sigma(1 / 2)}{g_{1}(1 / 2)}+\frac{\partial_{u} \sigma(-1 / 2)}{g_{1}(-1 / 2)}\right)
$$

where

$$
\overline{V_{m}}:=\frac{\left(v_{+}+v_{-}\right) \eta}{2 \chi^{*} L_{0}}
$$


Now, if we assume that the two parameters $\left(\overline{V_{m}}, \overline{\Delta V}\right)$, characterizing actin treadmilling, are fixed we can pose the optimization problem of finding the controls $g_{1}(u), g_{2}(u)$ ensuring the maximization of the normalized velocity $\bar{V}$. This problem is nontrivial because the functional $\bar{V}\left\{g_{1}, g_{2}\right\}$ is prescribed implicitly through the unknown solution of the boundary value problem $(6,8)$. To our advantage though this linear elliptic problem is classical and is well understood, e.g. [54].

We observe that parameter $\overline{V_{m}}$ enters the expression for the velocity (9) in an additive way and does not affect the solution of the optimization problem. The reason is that $\overline{V_{m}}$ characterizes a propulsion mode associated with simple accretion of the material at the front and its simultaneous removal at the rear; when $\overline{V_{m}} \neq 0$ an a priori polarity is imposed and the problem of motility initiation disappears. In view of the complete decoupling of this mode of self-propulsion from our controls, in what follows we assume without loss of generality that $\overline{V_{m}}=0$.

The second parameter $\overline{\Delta V}$, also characterizing the protrusion strength, does not induce polarity. As we clarify in the next Section, this parameter represents the mechanical action of pushers and the dependence of the crawling velocity on $\overline{\Delta V}$, which is now sensitive to both controls, is much more subtle than in the case of $\overline{V_{m}}$.

\section{Pushers and pullers}

To see that parameter $\overline{\Delta V}$ characterizes in our setting the activity of pushers, consider the global balance of couples in the co-moving coordinate system

$$
L \int_{-1 / 2}^{1 / 2} g_{1}(u) v(u) u d u=-\overline{\Delta V}+\int_{-1 / 2}^{1 / 2} g_{2}(u) d u+\sigma_{0} .
$$

Here the term in the left hand side characterizes the total moment due to external (frictional) forces. The first term in the the right hand side

$$
T=\overline{\Delta V}
$$

is due to active protrusion, while the second term $\int_{-1 / 2}^{1 / 2} g_{2}(u) d u=1$ is due to active contraction. The last term $\sigma_{0}$ corresponds to passive reaction forces resulting from the prescription of the length of the segment.

Our assumption that $\overline{\Delta V}>0$ means that the protrusion couple has a negative sign showing that the corresponding force dipoles act on the surrounding medium by pushing outward and creating negative stress. Instead, the contraction couple has a positive sign because the contractile forces pull inward and the induced stresses are positive. We can therefore associate protrusion with the presence of pushers and contraction with the activity of pullers [18, 21]. We can also (tentatively) argue that motility is protrusion-dominated when $T>1$ and it is contraction-dominated when $0<T<1$. This assertion will be confirmed later in the paper by rigorous analysis.

To illustrate the different roles played in our motility mechanism by pushers and pullers, we present below an analysis of a toy model which anticipates the main conclusions of the paper. We temporarily set $\overline{\Delta V}=0$ and describe the distribution of pushers and pullers by the same function $g_{2}(u)$ allowing it now to be both positive and negative. Our goal is to show that for protrusion-dominated motility driven by pushers, it is beneficial to create strong adhesion at the leading edge while for contraction-dominated motility driven by pullers, it is the trailing edge that has to adhere most strongly.

Consider a special choices of control functions,

$$
g_{1}(u)=q \delta\left(u-u_{1}\right)+(1-q) \delta\left(u-u_{2}\right), g_{2}(u)=p \delta\left(u-u_{3}\right)-(1-p) \delta\left(u-u_{4}\right),
$$

where $\delta$ is the Dirac distribution, $0 \leq q, p \leq 1$ and $-1 / 2 \leq u_{1}, u_{2}, u_{3}, u_{4} \leq 1 / 2$. The control function $g_{1}(u)$ represents two adhesion sites $u=u_{1}$ and $u=u_{2}$ whose locations and intensities must be optimized in relation to the prescribed position of a single puller placed at $u=u_{3}$ and characterized by a positive dipole moment $p$ and a single pusher located at $u=u_{4}$ and characterized by a negative dipole moment $p-1$. The parameter $p$ measures the relative importance of contraction comparing to protrusion.

Suppose that

$$
u_{3}<u_{1}, u_{2}<u_{4}
$$


which ensures that our active segment moves from left to right and that in the segment the adhesion sites are always outside the location of the active agents. By using (11) we can express the velocity of the segment as

$$
\bar{V}=\frac{1}{2}\left(1+\frac{(1-2 p)\left(u_{2}-u_{1}\right) c}{u_{1}-u_{2}+1+\left(u_{2}-u_{1}\right) a}\right)
$$

where,

$$
a=\frac{1}{1+q(1-q)\left(u_{2}-u_{1}\right) L^{2}} \text { and } c=\frac{1-2 q}{1+q(1-q)\left(u_{2}-u_{1}\right) L^{2}} .
$$

Suppose for simplicity that our two adhesive complexes are placed symmetrically with respect to the center of the segment $u_{1}=1-u_{2}$. We can then use a single parameter $\Delta u=u_{1}+1 / 2=1 / 2-u_{2}$ to obtain,

$$
\bar{V}=\frac{1}{2}\left(1+\frac{(1-2 p)(1-2 q)}{1+2 \Delta u q(1-q) L^{2}+\frac{2 \Delta u}{1-2 \Delta u}}\right) .
$$

At $\Delta u=0$, when adhesive complexes localize at the edges of the segment, the velocity reaches its maximum value

$$
\bar{V}=\frac{1}{2}[1+(1-2 p)(1-2 q)] .
$$

Because of the imposed inequalities (12), we must necessarily have in this configuration $u_{3}=-1 / 2$ and $u_{4}=1 / 2$ meaning that pushers and pullers must also localize at the edges. Notice however that inequalities $u_{3}<u_{1}$ and $u_{2}<u_{4}$ are necessary for formula (13) to hold so the apparent conclusion that $u_{3}=u_{1}=1 / 2$ and $u_{4}=u_{2}=-1 / 2$ is a result of an abuse of notation. In a theory where, pushers, pullers and adhesion complexes have a characteristic size of dispersion, the adhesion clusters will be slightly ahead of pullers and slightly behind the pushers so that active agents take advantage of the firm attachment to either push or pull (see more on this subject below).

From our simple analysis it follows that if pullers dominate $(p>1 / 2)$, the optimal way to tune adhesion is to set $q=1$ and concentrate adhesive complexes at the trailing edge of the moving segment achieving maximum velocity $\bar{V}=1$ when $p=1$. If, instead, pushers dominate $(p<1 / 2)$, the optimal way to tune adhesion is to set $q=0$ and concentrate adhesive complexes at the leading edge. In this case, the maximal velocity is again $\bar{V}=1$ given that $p=0$. In other words, to be effective, pullers have to concentrate on the trailing edge and ensure strong adhesion on the leading edge: in this way pullers can inflict contraction that displaces the trailing edge which due to the length constraint also propels the leading edge. On the contrary, pushers can take advantage of the firm attachment at the trailing edge to push against it and propel the leading edge which in turn pulls the trailing edge due to the length constraint.

\section{Contraction driven motility}

We now return to the study of the optimization problem in the original formulation. The simplest analytically transparent case is when protrusion is disabled $\overline{\Delta V}=0$ and motility is fully contraction-driven.

Suppose first that $g_{1} \equiv 1$ which means that the adhesion complexes are distributed uniformly. Then the velocity of the active segment can be expressed as a quadrature

$$
\bar{V}=-\frac{1}{2 \sinh \left(\frac{L}{2}\right)} \int_{-1 / 2}^{1 / 2} \sinh (L u) g_{2}(u) d u .
$$

One can see that if the function $g_{2}(u)$ is even, then $\bar{V}=0$. This result can be interpreted as an analogue of the famous Purcell's theorem about the necessity of non-symmetric strokes in Stokes swimming [4, 2]. If the distribution $g_{2}(u)$ is non-symmetric and, for instance, more motors are placed at the rear of the segment, the velocity will become positive. Using the fact $g_{2}(u) \geq 0$ we can also conclude from (14) that $\bar{V} \leq 1 / 2$. This upper bound is reached when all the motors are fully localized at the rear and $g_{2}(u)=\delta(u+1 / 2)$. 
Now, consider the general case when the focal adhesions are distributed inhomogeneously. Since (6) is a SturmLiouville problem, its solution can be written as

$$
\sigma(u)=\sigma_{0}-\int_{-1 / 2}^{1 / 2} G(u, s)\left[g_{2}(s)-\sigma_{0}\right] d s,
$$

where the Green's function $G(u, s)$ can be represented two auxiliary functions $h(u)$ and $f(u)$

$$
G(u, s)=\frac{1}{C}\left[h(u) f(s) \nVdash_{[s<u]}+h(s) f(u) \nVdash_{[u<s]}\right],
$$

solving the following standard boundary value problems [54] :

$$
\left\{\begin{array}{c}
\left(\frac{1}{g_{1}} h^{\prime}\right)^{\prime}=L^{2} h \\
h(-1 / 2)=1, h(1 / 2)=1
\end{array},\left\{\begin{array}{c}
\left(\frac{1}{g_{1}} f^{\prime}\right)^{\prime}=L^{2} f \\
f(-1 / 2)=1, f(1 / 2)=-1
\end{array} .\right.\right.
$$

In (16), $C=\left(h f^{\prime}-f h^{\prime}\right) / g_{1}$ is a constant involving the Wronskian of the two auxiliary functions $h(u)$ and $f(u)$ and $\nVdash$ is the indicator function. We can now write

$$
\bar{V}=\frac{1}{2} \int_{-1 / 2}^{1 / 2} f(u)\left(g_{2}(u)-\hat{g}_{2}\right) d u,
$$

where we introduced a new measure of inhomogeneity of contraction:

$$
\hat{g}_{2}=\frac{\int_{-1 / 2}^{1 / 2} h(u) g_{2}(u) d u}{\int_{-1 / 2}^{1 / 2} h(u) d u}
$$

If both functions $g_{1,2}(u)$ are even, then $f(u)$ is odd and, since the integral of a product of an odd and an even functions is equal to zero, we obtain that $\bar{V}=0$. The same result follows if we assume that contraction is homogeneous $g_{2}(u)=\hat{g}_{2}=1$ while the adhesion distribution $g_{1}(u)$ is arbitrary. Therefore, to ensure motility at $\overline{\Delta V}=0$, contraction must be inhomogeneous while adhesion may still be uniform provided contraction is not even.

To find the optimal distributions $g_{1}(u), g_{2}(u)$ we proceed in two steps. We first show that $\bar{V} \leq 1$ and then find a configuration of controls allowing the cell to reach this bound.

Notice that we can rewrite (18) in the form

$$
\bar{V}=\frac{1}{2}\left(\int_{S_{+}} f(u)\left(g_{2}(u)-\hat{g}_{2}\right) d u+\int_{S_{-}} f(u)\left(g_{2}(u)-\hat{g}_{2}\right) d u\right)
$$

where we defined the domains $S_{-}=\left\{u / g_{2}(u) \leq \hat{g}_{2}\right\}$ and $S_{+}=\left\{u / g_{2}(u)>\hat{g}_{2}\right\}$. Applying the maximum principle to (17) we obtain that $1 \geq h(u) \geq 0$ and $h(u) \geq f(u) \geq-h(u)$. Using the bounds on $f$, we can write

$$
\bar{V} \leq \frac{1}{2}\left(\int_{S_{+}} h(u) g_{2}(u) d u+\hat{g}_{2} \int_{S_{-}} h(u) d u\right) .
$$

Since the integrands are positive and $h(u) \leq 1$ it finally follows that

$$
\bar{V} \leq \int_{-1 / 2}^{1 / 2} h(u) g_{2}(u) d u \leq \int_{-1 / 2}^{1 / 2} g_{2}(u) d u=1 .
$$

Observe that in the case of a homogeneous distribution of adhesive clusters, the velocity could reach only one half of this maximal value.

We now show that the maximal velocity $\bar{V}=1$ can be reached if both controls $g_{1}(u)$ and $g_{2}(u)$ are fully localized. Take $\theta>0$ and consider a regularized distribution

$$
g_{1}(u ; \theta)=\frac{1}{\pi} \frac{\theta}{\theta^{2}+\left(u-u_{1}\right)^{2}} .
$$


For this choice of $g_{1}(u)$ the auxiliary functions $h(u)$ and $f(u)$ can be written explicitly in term of Legendre polynomials. In the limit $\theta \rightarrow 0$ and $\lim _{\theta \rightarrow 0} g_{1}(u ; \theta)=\delta\left(u-u_{1}\right)$ we obtain

$$
h(u)=1 \text { and } f(u)=\left\{\begin{array}{c}
1 \text { if } u \leq u_{1} \\
-1 \text { if } u>u_{1} .
\end{array}\right.
$$

By using these explicit expressions for the auxiliary functions we can rewrite (18) in the form

$$
\bar{V}=\frac{1}{2}\left[\int_{-1 / 2}^{u_{1}} g_{2}(u) d u-\int_{u_{1}}^{1 / 2} g_{2}(u) d u-2 u_{1}\right] .
$$

if we now suppose that $g_{2}(u)=\delta\left(u-u_{2}\right)$ the expression for velocity reduces to reduces to

$$
\bar{V}=\frac{1}{2}\left\{\begin{array}{c}
1-2 u_{1} \text { if } u_{2}<u_{1} \\
-2 u_{1} \text { if } u_{2}=u_{1} \\
-1-2 u_{1} \text { if } u_{2}>u_{1}
\end{array}\right.
$$

It is now clear that the velocity reaches its maximal value as $u_{1} \rightarrow-1 / 2$ while $u_{2}<u_{1}$. We can then formally write $u_{2}=u_{1}=-1 / 2$ and obtain the controls $g_{2}(u)=g_{1}(u)=\delta(u+1 / 2)$ saturating the bound $\bar{V}=1$. Notice, however, that if we assume directly $u_{1}=u_{2} \rightarrow-1 / 2$ in (20), we obtain $\bar{V}=1 / 2$. This is in agreement with the physical intuition that the anchorage point must be located to the right of the pulling force dipole: in this case the pulling forces advance the rear edge of the segment with minimal slipping. Mathematically, we encounter here the case of non-commutation of the limiting procedures $u_{2} \rightarrow-1 / 2$ and $u_{1} \rightarrow-1 / 2$ giving $\bar{V}=1$ only if the limits are taken in the above order.

To summarize, the optimization of the distribution of focal adhesions allows the contraction-dominated mechanism of cell motility to reach the value of velocity which is twice as large as when the adhesion is uniform. This means that in order to improve the motility performance the adhesion machinery must conspire with the contraction machinery making sure that both the motors and the adhesive centers are localized at the trailing edge. Interestingly, exactly this type of correlation between the stresses created by contraction and the distribution of focal adhesions was observed in experiments and numerical simulations [55, 56, 57, 58, 59, 22]. The localization of adhesion complexes close to cell edges, where contraction is the strongest, has been also reported outside the motility context [60, 61, 62].

\section{Upper and lower bounds for velocity in the general case}

We now turn to the general case where both contraction and protrusion are active. In particular, the protrusive power will be characterized by the parameter $\overline{\Delta V}=T>0$ which was assumed to be equal to zero in the previous Section. We can then write

$$
\bar{V}=\frac{1}{2}\left[\frac{\int_{-1 / 2}^{1 / 2} f(u) d u}{\int_{-1 / 2}^{1 / 2} h(u) d u} T+\int_{-1 / 2}^{1 / 2} f(u)\left(g_{2}(u)-\hat{g}_{2}\right) d u\right] .
$$

As we see, the first term in the right hand side is associated with protrusion-based (or filament-driven [63]) motility while, as we have already seen, the second term is the contribution due to contraction-based (or motor-driven [63] ) motility. We notice that if $g_{1}(u)$ is even, then $f(u)$ is odd and $h(u)$ is even, leading to

$$
\frac{\int_{-1 / 2}^{1 / 2} f(u) d u}{\int_{-1 / 2}^{1 / 2} h(u) d u}=0
$$

If $g_{2}(u)$ is also even, then

$$
\int_{-1 / 2}^{1 / 2} f(u)\left(g_{2}(u)-\hat{g}_{2}\right) d u=0 .
$$

In this case the velocity of the segment is fully controlled by the accretion mechanism characterized by the parameter $\overline{V_{m}}$. 
Consider first the case of protrusion-driven motility by assuming that contraction is homogeneous $g_{2}(u) \equiv 1$ and therefore does not contribute to the overall velocity. By using again the maximum principle we obtain inequalities

$$
-1 \leq \frac{\int_{-1 / 2}^{1 / 2} f(u) d u}{\int_{-1 / 2}^{1 / 2} h(u) d u} \leq 1,
$$

leading to the upper bound

$$
\bar{V}=\overline{V_{p}} \leq \frac{T}{2} .
$$

The maximum of the protrusive contribution to velocity is reached when, $g_{1}(u)=\delta\left(u-\frac{1}{2}\right)$, because in this case $h=1$ and $f=1$ almost everywhere. Observe, that the optimal solution in the case of protrusion-driven motility is in some sense opposite to the solution $g_{1}(u)=\delta(u+1 / 2)$ obtained in the case of the contraction-driven motility.

Based on (19) and (22) we can now argue that in the case when both treadmilling and contraction are present, an upper bound for velocity is

$$
\bar{V} \leq \frac{T}{2}+1
$$

however, as we have just seen, in view of the incompatibility of the corresponding optimal controls, this bound cannot be reached. The optimal strategy for focal adhesions would then involve a compromise between the necessity to localize adhesion at the trailing edge in order to assist the contraction mechanism and the competing trend to localize adhesion at the leading edge in order to improve the protrusion power of the cell.

To obtain a lower bound for $\bar{V}$ we now consider a particular test function representing a weighted sum of our competing optimal controls, $g_{1}(u)=q \delta(u+1 / 2)+(1-q) \delta(u-1 / 2)$. We also chose $g_{2}(u)=\delta\left(u-u_{0}\right)$, where $q \in[0,1]$ and $u_{0} \in[-1 / 2,1 / 2]$ are two parameters to be optimized. Then, by solving (17) we obtain,

$$
f(u)=\left\{\begin{array}{c}
1 \text { if } u=-1 / 2 \\
\left.\frac{1-2 q}{1+q(1-q) L^{2}} \text { if } u \in\right]-1 / 2,1 / 2[ \\
-1 \text { if } u=1 / 2
\end{array}\right.
$$

and,

$$
h(u)=\left\{\begin{aligned}
1 & \text { if } u=-1 / 2 \\
\frac{1}{1+q(1-q) L^{2}} & \text { if } u \in]-1 / 2,1 / 2[ \\
1 & \text { if } u=1 / 2,
\end{aligned}\right.
$$

which leads to the expression for the velocity

$$
\bar{V}=\frac{T}{2}(1-2 q)+\frac{1}{2}\left(f\left(u_{0}\right)-(1-2 q) h\left(u_{0}\right)\right) .
$$

The optimization with respect to $u_{0}$ gives $u_{0}=-1 / 2$ and

$$
\bar{V}=\frac{T}{2}-q(T-1)
$$

Finally, optimizing in $q$ we obtain that if $T<1$, we need to take $q=0$ and if $T>1$, we get $q=1$. This result, illustrated in Fig 1, suggests that there is a switch at $T=1$ between the contraction-centered optimization strategy $(q=0)$ and the protrusion-centered optimization strategy $(q=1)$. Notice that the switch takes place exactly when the negative protrusion generated couple $T$ becomes equal to the positive contractile couple equal to 1 . At an interesting state $T=$ 1 , the two active mechanisms neutralize each other and activity-related dipoles become invisible behind the passive contributions in Eq. (10): in this case the optimal position of active and adhesive agents becomes indeterminate. 


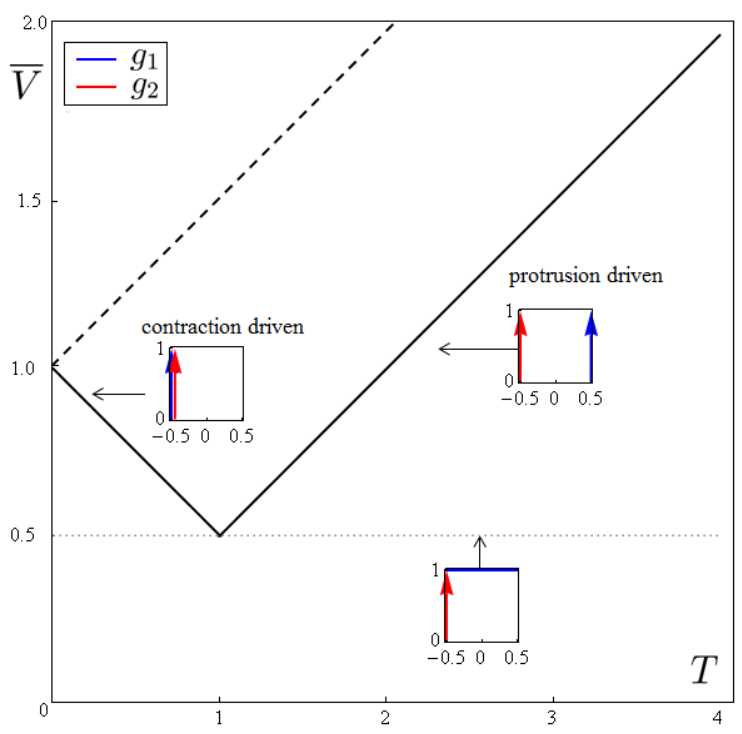

Figure 1: Solid lines: Lower bound on the optimal velocity of self-propulsion $\bar{V}$ as a function of the measure of the (relative) protrusive strength $T$. The optimal strategy depends on whether contraction $(T<1)$ or protrusion $(T>1)$ dominates. The dashed line represents the upper bound obtained by formally summing the incompatible upper bounds for the protrusion and contraction based strategies. The dotted line represents a sub-optimal strategy obtained under the assumption that adhesion is homogeneous. Insets illustrate the associated configurations of controls $g_{1}(u)$ and $g_{2}(u)$.

\section{Numerical solution of the optimization problem}

To show that the low bound obtained in the previous Section is rather close to being optimal, here we solve the optimization problem numerically. A finite dimensional reduction of the original variational problem is constructed by selecting $N+2$ points $u_{i}=i /(N+1)-1 / 2$ that subdivide the segment $[-1 / 2,1 / 2]$. We then localize adhesion and contraction in these points by choosing the control functions in the form

$$
g_{1}(u)=\sum_{i=1}^{N} g_{1}^{i} \delta\left(u-u_{i}\right), g_{2}(u)=\sum_{i=0}^{N+1} g_{2}^{i} \delta\left(u-u_{i}\right)
$$

where $\sum_{i=0}^{N+1} g_{2}^{i}=\sum_{i=1}^{N} g_{1}^{i}=1$ and $g_{1}^{i} \geq 0, g_{2}^{i} \geq 0$. In this way we also mimic the discrete nature of real adhesive clusters and real myosin motors, so in some respects the discrete problem is even more realistic than the continuum one.

By solving the auxiliary linear elliptic problems for this choice of controls we obtain that the functions $h(u)$ and $f(u)$ are piece-wise constant $h(u)=\sum_{i=0}^{N} A_{i} \nVdash_{\left[u_{i}, u_{i+1}[\right.}(u)$ and $f(u)=\sum_{i=0}^{N} C_{i} \nVdash_{\left[u_{i}, u_{i+1}[\right.}(u)$. The coefficients with $i \in[2, N]$ satisfy the equations

$$
\begin{aligned}
& g_{1}^{i}\left(A_{i-1}-A_{i-2}\right)+g_{1}^{i} g_{1}^{i-1} A_{i-1} L^{2}\left(u_{i}-u_{i-1}\right)=g_{1}^{i-1}\left(A_{i}-A_{i-1}\right) \\
& g_{1}^{i}\left(C_{i-1}-C_{i-2}\right)+g_{1}^{i} g_{1}^{i-1} C_{i-1} L^{2}\left(u_{i}-u_{i-1}\right)=g_{1}^{i-1}\left(C_{i}-C_{i-1}\right)
\end{aligned}
$$

The boundary conditions give

$$
A_{0}=1, A_{N}=1, C_{0}=1, C_{N}=-1 \text {. }
$$

We use the conventions $A_{-1}=A_{0}, A_{N+1}=A_{N}, C_{-1}=C_{0}$ and $C_{N+1}=C_{N}$ to express the velocity $V$ in the form

$$
\bar{V}=\frac{1}{2}\left(T-\sum_{i=0}^{N+1} g_{2}^{i} \frac{A_{i}+A_{i-1}}{2}\right) \frac{\sum_{i=0}^{N} C_{i}\left(u_{i+1}-u_{i}\right)}{\sum_{i=0}^{N} A_{i}\left(u_{i+1}-u_{i}\right)}+\frac{1}{2} \sum_{i=0}^{N+1} g_{2}^{i} \frac{C_{i}+C_{i-1}}{2} .
$$


The function (23) was optimized numerically with respect to parameters $g_{1}^{i}$ and $g_{2}^{i}$ subjected to the appropriate equality and inequality type constraints. To find the global minimum we used the method of simulated annealing with initial guesses corresponding to homogeneous configurations. In Fig.2, illustrating our results for $N=100$, one can see that for $T<1$ both optimal functions $g_{1}(u)$ and $g_{2}(u)$ are localized at the trailing edge. Instead, for $T>1$ we observe that $g_{1}(u)$ localizes at the leading edge while $g_{2}(u)$ localizes at the trailing edge. The value of the maximal velocity, obtained numerically, is the same as in the bound up to an error proportional to the mesh size. Notice also that the optimally spaced adhesion points are shifted from the locations of force dipoles by a mesh size. Thus, the solution of the numerical (regularized) problem in the contraction-dominated regime (with $T=0.9$ ), shown in Fig. 2, clearly distinguishes the optimal functions $g_{1}(u)$ and $g_{2}(u)$ that are both localized at the size of the mesh. The function $g_{1}(u)$ remains different from zero at one mesh size beyond the point where we already have $g_{2}(u)=0$ (for positive velocity). In the protrusion-dominated regime (at $T=1.1$ ) shown in Fig. 2, the mesh size again prevents localization of the function $g_{1}(u)$ exactly at the leading edge while $g_{2}(u)$ localizes exactly at the trailing edge. These observations confirm that the regularized numerical solution is more realistic than its singular analytic prototype. Overall, our numerical results are in full agreement with the analytic bounds which suggests that that these bounds are (nearly) sharp.
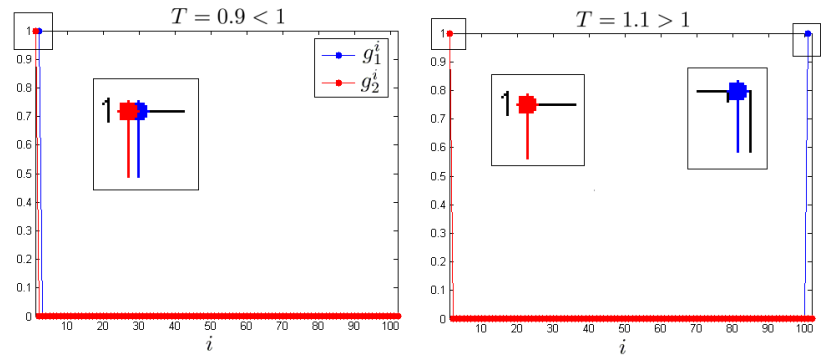

Figure 2: Numerical results for the optimization of the function (23) for two values of the protrusive strength, below the threshold $T=0.9$ and above the threshold $T=1$.1. Insets magnify the structure of the optimal controls near the boundaries of the moving segment. Parameter $L=10$.

\section{Local stability analysis}

In this Section we use a perturbation analysis to provide additional evidence that our lower bounds are close to being optimal.

In what follows we use the superscript $\circ$ to indicate the unperturbed state and superscript $\star$ to mark parameters characterizing the perturbed configuration. We assume that for all functions $\phi(u)$ the following expansion holds in the first order

$$
\phi(u)=\phi^{\circ}(u)+\epsilon \phi^{\star}(u),
$$

where $\epsilon$ is a small parameter. Keeping only the first order term in the expression for $\bar{V}$, we obtain

$$
\begin{aligned}
\bar{V}^{\star}= & \frac{1}{2}\left[\int_{-1 / 2}^{1 / 2} g_{2}^{\circ} f^{\star}+f^{\circ} g_{2}^{\star}-\frac{\int_{-1 / 2}^{1 / 2} f^{\circ}}{\int_{-1 / 2}^{1 / 2} h^{\circ}}\left(\int_{-1 / 2}^{1 / 2} g_{2}^{\circ} h^{\star}+h^{\circ} g_{2}^{\star}\right)\right. \\
& \left.+\frac{T-\int_{-1 / 2}^{1 / 2} h^{\circ} g_{2}^{\circ}}{\int_{-1 / 2}^{1 / 2} h^{\circ}}\left(\int_{-1 / 2}^{1 / 2} f^{\star}-\frac{\int_{-1 / 2}^{1 / 2} h^{\star}}{\int_{-1 / 2}^{1 / 2} h^{\circ}} \int_{-1 / 2}^{1 / 2} f^{\circ}\right)\right],
\end{aligned}
$$

where $h^{\star}=H^{\star \prime}, f^{\star}=F^{\star \prime}$ and

$$
\left\{\begin{array}{c}
H^{\star \prime \prime}-L^{2} g_{1}^{\circ} H^{\star}=L^{2} h^{\circ} g_{1}^{\star} \\
H^{\star \prime}(-1 / 2)=H^{\star \prime}(1 / 2)=0
\end{array},\left\{\begin{array}{c}
F^{\star \prime \prime}-L^{2} g_{1}^{\circ} F^{\star}=L^{2} F^{\circ} g_{1}^{\star} \\
F^{\star \prime}(-1 / 2)=F^{\star \prime}(1 / 2)=0 .
\end{array}\right.\right.
$$

In view of the saturation of the constraints by the unperturbed solution $\int_{-1 / 2}^{1 / 2} g_{2}^{\circ}=\int_{-1 / 2}^{1 / 2} g_{1}^{\circ}=1$, we must have

$$
\int_{-1 / 2}^{1 / 2} g_{2}^{\star}=\int_{-1 / 2}^{1 / 2} g_{1}^{\star}=0
$$


Suppose now that we perturb the 'optimal' controls $g_{1}^{\circ}(u)=g_{2}^{\circ}(u)=\delta(u+1 / 2)$ delivering the lower bound for velocity at $T<1$. We can again formally set

$$
h^{\circ}(u) \equiv 1 \text { and } f^{\circ}(u)=\left\{\begin{array}{c}
1 \text { if } u=-1 / 2 \\
-1 \text { if } u>-1 / 2,
\end{array}\right.
$$

while remembering that the localization point for adhesion must be shifted with respect to the point of localization of contraction. The perturbation of velocity can be written as

$$
\bar{V}^{\star}=\frac{1}{2}\left[\int_{-1 / 2}^{1 / 2}\left(f^{\circ}(u)+h^{\circ}(u)\right) g_{2}^{\star}(u) d u+(T-1) \int_{-1 / 2}^{1 / 2}\left(f^{\star}(u)+h^{\star}(u)\right) d u\right] .
$$

A rather general class of perturbed controls can be represented in the form

$$
\left\{\begin{array}{l}
g_{1}^{\star}(u)=-q \delta\left(u+\frac{1}{2}\right)+r(u) \text { with, } \int_{-1 / 2}^{1 / 2} r(u) d u=q \\
g_{2}^{\star}(u)=-p \delta\left(u+\frac{1}{2}\right)+l(u) \text { with, } \int_{-1 / 2}^{1 / 2} l(u) d u=p,
\end{array}\right.
$$

Since $g_{1} \geq 0$ and $g_{2} \geq 0$ we demand that $r(u) \geq 0$ and $l(u) \geq 0$ and therefore also $q \geq 0$ and $p \geq 0$. We obtain

$$
\bar{V}^{\star}=-p+(T-1) \int_{-1 / 2}^{1 / 2} r(u)(u+1 / 2) d u .
$$

From (26) we see that if $T \leq 1$, a perturbation of the controls $g_{1}^{\circ}(u)=g_{2}^{\circ}(u)=\delta(u+1 / 2)$ leads to the decrease of the velocity: $\bar{V}^{\star} \leq 0$. Instead, if $T>1$, by choosing $r(u)$ such that,

$$
\int_{-1 / 2}^{1 / 2} r(u)\left(u+\frac{1}{2}\right) d u>\frac{p}{T-1},
$$

we obtain a perturbation with $\bar{V}^{\star}>0$. These observations suggest that beyond the threshold $T=1$ the control function $g_{1}(u)$ should be no longer localized at the trailing edge edge. We also see that the most 'efficient' way to make the velocity larger at $T>1$ is to localize the function $r(u)$ at the leading edge of the segment (at $u=1 / 2)$.

Consider now a perturbation of the set of controls $g_{1}^{\circ}(u)=\delta(u-1 / 2)$ and $g_{2}^{\circ}(u)=\delta(u+1 / 2)$ delivering our lower bound for $T>1$. With the same abuse of notations as before we can write

$$
h^{\circ}(u) \equiv 1 \text { and } f^{\circ}(u)=\left\{\begin{array}{c}
1 \text { if } u<1 / 2 \\
-1 \text { if } u=1 / 2 .
\end{array}\right.
$$

We represent the perturbations in the form

$$
\left\{\begin{array}{c}
g_{1}^{\star}(u)=-q \delta\left(u-\frac{1}{2}\right)+r(u) \text { with, } \int_{-1 / 2}^{1 / 2} r(u) d u=q \geq 0 \\
g_{2}^{\star}(u)=-p \delta\left(u+\frac{1}{2}\right)+l(u) \text { with, } \int_{-1 / 2}^{1 / 2} l(u) d u=p \geq 0 .
\end{array}\right.
$$

where again $r(u) \geq 0$ and $l(u) \geq 0$ and therefore also $q \geq 0$ and $p \geq 0$. The ensuing perturbation of velocity is

$$
\bar{V}^{\star}=\frac{1}{2}\left[\int_{-1 / 2}^{1 / 2}\left(f^{\circ}(u)-h^{\circ}(u)\right) g_{2}^{\star}(u) d u+(T-1) \int_{-1 / 2}^{1 / 2}\left(f^{\star}(u)-h^{\star}(u)\right) d u\right]=-(T-1) \int_{-1 / 2}^{1 / 2}\left(\frac{1}{2}-u\right) r(u) d u
$$

It is now clear that if $T \geq 1$, then $\bar{V}^{\star} \leq 0$, showing that the perturbations of controls are sub-optimal. This gives another evidence that the test function providing the lower bound for velocity at $T \geq 1$ are at least close to being optimal.

Based on this analysis we conjecture that the function $\bar{V}(T)$, representing the optimal velocity, is piece-wise linear with a kink at $T=1$. The presence of a threshold indicates a switch from contraction-dominated motility pattern to protrusion-dominated motility pattern. As the relative power of protrusion, epitomized by $T$, increases beyond this threshold, the focal adhesions, maintaining the optimality of the self-propulsion velocity, must migrate from the trailing to the leading edge of the active segment. The dynamic migration of adhesion proteins to the edges has been observed in experiments [60]. In real cells, however, both edges are usually populated by adhesion complexes and we can speculate that in this way cells can adjust smoothly to transitions from one driving mode to another. 


\section{Discussion}

In this paper we used a simple analytically tractable model of cell motility to study the optimal strategies allowing cells to move faster by actively coordinating spatial distributions of contractile and adhesive agents. Our study reveals that if adhesion complexes can detect the dominating mechanism of self propulsion, they can self-organize to ensure the best performance.

We made specific predictions regarding the advantageous correlations between the distributions of adhesive and force producing agents and showed that the dependence of the maximal velocity of self-propulsion on the relative strength of contraction and protrusion may be non-monotone. In particular, our model predicts that a limited activation of protrusion will necessarily lower the maximal velocity achieved in a purely contractile mode of self-propulsion. However, as the protrusion strength increases, protrusion can overtake contraction and the velocity of self-propulsion will increase beyond the level achieved in the contraction-dominated case.

Previously we have shown that contraction-driven motility mechanism may be sufficient by itself to explain cell polarization, motility initiation, motility arrest and the symmetrization of a cell before mitosis [31, 44]. From the analysis presented in this paper it becomes evident that, if the speed of self propulsion is an issue, cells should mostly rely on protrusion. More specifically, to maximize its velocity performance after motility initiation a cell must switch from contraction-dominated to protrusion-dominated motility mechanism by increasing the protrusive power and appropriately rearranging the distribution of adhesive complexes. It was shown in [43] that similar transitions between contraction and protrusion mechanisms can be used by a cell to accommodate different types of cargos.

To compare our predictions with experiments we can use numerical values of parameters for keratocyte fragments $[29,34,32]$. We obtain the following rough estimates: $\chi^{*}=10^{3} \mathrm{~Pa}, \xi^{*}=3 \times 10^{16} \mathrm{~Pa} \cdot \mathrm{m}^{-2} \cdot s, \eta=3 \times 10^{4} \mathrm{~Pa} \cdot \mathrm{s}$, $L_{0}=10 \mu m, V_{m}=8 \mu m \cdot \mathrm{min}^{-1}$ and $\Delta V=0.6 \mu \mathrm{m} \cdot \mathrm{min}^{-1}$. Our first quantitative prediction concerns the case when active treadmilling of actin is knocked down and adhesion is homogeneous. As we have shown, the largest velocity in this case is reached when all myosin motors are localized at the trailing edge as observed in most eukariotic cells $[64,65,66,67]$. In dimensional form, the predicted maximal velocity is $V=L_{0} \chi^{*} /(2 \eta) \approx 10 \mu m \cdot \mathrm{min}^{-1}$, which is low in view of the data on keratocyte fragments suggesting that velocity should be in the interval $30-40 \mu \mathrm{m} \cdot \mathrm{min}^{-1}$ [68]. This is not surprising because according to our results only half of the total amount of motors is "used" in this case which implies that adhesion homogeneity is highly sub-optimal.

If the adhesion inhomogeneity is allowed, the configuration becomes optimal when both myosins and integrins are localized at the trailing edge. Such highly correlated distributions have been observed in experiments and generated in microscale-based numerical models $[60,56,61,62,57,58,59,69]$. In this case, as a result of the cooperative response, a cell can be more efficient achieving the velocity that is two times larger than in the case of homogeneous adhesion: $V=L_{0} \chi^{*} / \eta \approx 20 \mu m \cdot \mathrm{min}^{-1}$.

To be even closer to reality we need to take active treadmilling into consideration and our estimates suggest that $T \approx 0.1 \ll 1$. This means that we are in the contraction-dominated motility regime for which our velocity bound gives more realistic value $V=V_{m}+L_{0} \chi^{*} / \eta \approx 28 \mu m \cdot \mathrm{min}^{-1}$. Notice, however, that reducing the value $\chi^{*}$ by one order of magnitude, which is within the existing error bounds, we may easily reach the regime where $T>1$ and where it would becomes more beneficial for adhesion clusters to localize at the leading edge conspiring with protrusive elements. A spatial correlation of this type between adhesion and protrusion has been recorded in both experiments and comprehensive numerical models, see for instance the data in [70, 71] on nematode spermatozoa.

The proposed model can be also tested indirectly. For instance, we know that the location of adhesion complexes in a moving cell can be identified by measuring the distribution of traction forces in the elastic environment $[72,73]$. If the adhesive complexes are found to be shifted towards the leading edge, we would argue that the cell relies for its advance mostly on actin treadmilling. If instead the adhesive complexes are preferentially positioned at the trailing edge, our model suggests that motility is mostly driven by contraction. Both predictions can be tested by independent measurements.

An interesting possibility would be if cells could alternate the location of maximum adhesion between the trailing edge and the leading edge in response to oscillations in the level of activity of pullers and pushers. The evidence of such switching may be that both location are populated with adhesive complexes. We may also recall that the classical mechanism of crawling for eukaryotic cells involves two phases [24, 74, 26, 75]: one of them is associated with the creation of protrusions that push at the front while relying on the stabilization of the trailing edge and another one involves pulling of the rear (of the cargo) which requires fixation at the front. The switching between these two phases 
takes place almost periodically and the associated reorganization of adhesion clusters from the leading to the trailing edge has been well documented [76, 72]. To capture such non-steady motility pattern in the framework of our model, the simplifying traveling wave assumption would have to be replaced by a more complex ansatz.

In conclusion, we emphasize that our interpretations are based on the study of a variational problem whose analysis revealed some interesting correlations between the spatial arrangement of adhesion and contraction agents and has led us to quantitative predictions that are in agreement with experiment. The prototypical nature of the proposed model, however, conceals considerable complexity of the actual cell motility phenomenon which involves intricate bio-chemical feedback loops, geometrically complex mechanical flows and highly nontrivial rheological behavior. In particular, the singular nature of the obtained solutions can be at least partially linked to the fact that treadmilling is modeled schematically, with polymerization and depolymerization processes localized at the edges: at least one additional control function describing the distribution of pushers is needed to regularize the problem in this respect. The situation is complicated further by the fact that the dominant trade-off condition, controlling the self-organization of active agents, is still unknown notwithstanding some recent results in this direction [23]. However, even in the absence of the definitive optimization criterion and with minimal assumptions about the inner working of the motility machinery, our study reveals that depending on the task and the available resources a cell may have to modify its mode of operation rather drastically to ensure the best possible performance.

\section{Acknowledgments}

The authors are thankful to F. Alouges, D. Ambrosi, G. Geymonat and A. Zanzottera for helpful discussions. The work of P.R. was supported by Monge Doctoral Fellowship at Ecole Polytechnique.

\section{References}

\section{References}

[1] Childress S. Mechanics of swimming and flying. vol. 2. Cambridge University Press; 1981.

[2] Lauga E, Powers TR. The hydrodynamics of swimming microorganisms [Review]. Reports on Progress in Physics. 2009 SEP;72(9).

[3] Taylor G. Analysis of the swimming of microscopic organisms. Proceedings of the Royal Society of London Series A Mathematical and Physical Sciences. 1951;209(1099):447-461.

[4] Purcell EM. Life at low Reynolds number. American Journal of Physics. 1977;45(1):3-11. Available from: http://dx.doi.org/10. 1119/1.10903.

[5] DeSimone A, Alouges F, Lefebvre A. Biological Fluid Dynamics: Swimming at low Reynolds numbers. Preprint SISSA. $2008 ; 21: 1-13$.

[6] Shapere A, Wilczek F. Self-Propulsion at Low Reynolds Number. Phys Rev Lett. 1987 May;58:2051-2054.

[7] Najafi A, Golestanian R. Simple swimmer at low Reynolds number: Three linked spheres. Phys Rev E. 2004 Jun;69:062901. Available from: http://link.aps.org/doi/10.1103/PhysRevE.69.062901.

[8] Leshansky AM, Kenneth O, Gat O, Avron JE. A frictionless microswimmer. New Journal of Physics. 2007;9(5):145. Available from: http://stacks.iop.org/1367-2630/9/i=5/a=145.

[9] Alouges F, DeSimone A, Lefebvre A. Optimal strokes for axisymmetric microswimmers. The European Physical Journal E: Soft Matter and Biological Physics. 2009;28:279-284. Available from: http://dx.doi.org/10.1140/epje/i2008-10406-4.

[10] Alouges F, DeSimone A, Heltai L. Numerical strategies for stroke optimization of axisymmetric microswimmers. Mathematical Models and Methods in Applied Sciences. 2011;21(02):361-387.

[11] Osterman N, Vilfan A. Finding the ciliary beating pattern with optimal efficiency. Proceedings of the National Academy of Sciences of the United States of America. 2011;108(38):15727-15732.

[12] Michelin S, Lauga E. Unsteady feeding and optimal strokes of model ciliates. arXiv preprint arXiv:12101331. 2012;.

[13] DeSimone A, Tatone A. Crawling motility through the analysis of model locomotors: Two case studies. The European Physical Journal E. 2012;35:1-8. Available from: http://dx.doi.org/10.1140/epje/i2012-12085-x.

[14] Noselli G, Tatone A, DeSimone A. Discrete one-dimensional crawlers on viscous substrates: achievable net displacements and their energy cost. Mechanics Research Communications. 2014;58:73-81.

[15] Gidoni P, Noselli G, DeSimone A. Crawling on directional surfaces. International Journal of Non-Linear Mechanics. 2014;61:65-73.

[16] Mogilner A; European Soc Math \& Theoret Biol. Mathematics of cell motility: have we got its number? [Proceedings Paper]. Journal of Mathematical Biology. 2009 JAN;58(1-2):105-134. International Conference on Industrial and Applied Mathematics, Zurich, SWITZERLAND, 2007.

[17] Simha R, Ramaswamy S. Hydrodynamic fluctuations and instabilities in ordered suspensions of self-propelled particles. Physical Review Letters. 2002 JUL 29;89(5).

[18] Carlsson AE. Mechanisms of cell propulsion by active stresses. New Journal of Physics. 2011 JUL 7;13. 
[19] Saintillan D, Shelley MJ. Emergence of coherent structures and large-scale flows in motile suspensions. Journal of the Royal Society Interface. 2012 MAR 7;9(68):571-585.

[20] Marchetti MC, Joanny JF, Ramaswamy S, Liverpool TB, Prost J, Rao M, et al. Soft Active Matter. ArXiv e-prints. 2012 Jul;.

[21] Recho P, Putelat T, Truskinovsky L. Contraction-Driven Cell Motility. Phys Rev Lett. 2013;111:108102.

[22] Gao H, Qian J, Chen B. Probing mechanical principles of focal contacts in cell-matrix adhesion with a coupled stochastic-elastic modelling framework. Journal of the Royal Society Interface. 2011 SEP 7;8(62):1217-1232.

[23] Recho P, Joanny JF, Truskinovsky L. Optimality of contraction-driven crawling. Physical Review Letters. 2014;112(21):218101.

[24] Abercrombie M. Croonian lecture, 1978 - Crawling movement of metazoan cells [Article]. Proceedings of the Royal Society of London Series B-Biological Sciences. 1980;207(1167):129-\&.

[25] DiMilla P, Barbee K, Lauffenburger D. Mathematical-model for the effects of adhesion and mechanics on cell-migration speed [Article]. Biophysical journal. 1991 JUL;60(1):15-37.

[26] Stossel T. On the crawling of animal-cells [Article]. Science. 1993 May 21;260(5111):1086-1094.

[27] Ridley AJ, Schwartz MA, Burridge K, Firtel RA, Ginsberg MH, Borisy G, et al. Cell Migration: Integrating Signals from Front to Back. Science. 2003;302(5651):1704-1709. Available from: http://www.sciencemag.org/content/302/5651/1704.abstract.

[28] Vicente-Manzanares M, Webb D, Horwitz A. Cell migration at a glance [Article]. Journal of Cell Science. 2005 NOV 1;118(21).

[29] Kruse K, Joanny JF, Julicher F, Prost J. Contractility and retrograde flow in lamellipodium motion [Article]. Physical Biology. 2006 JUN;3(2):130-137.

[30] Hoffman BD, Crocker JC. Cell Mechanics: Dissecting the Physical Responses of Cells to Force. Annual Review of Biomedical Engineering. 2009;11:259-288.

[31] Recho P, Putelat T, Truskinovsky L. Contraction-Driven Cell Motility. Phys Rev Lett. 2013 Sep;111:108102. Available from: http: //link.aps.org/doi/10.1103/PhysRevLett.111.108102.

[32] Julicher F, Kruse K, Prost J, Joanny JF. Active behavior of the cytoskeleton [Review]. Physics Reports-Review Section of Physics Letters. 2007 SEP;449(1-3):3-28.

[33] Rubinstein B, Fournier MF, Jacobson K, Verkhovsky AB, Mogilner A. Actin-Myosin Viscoelastic Flow in the Keratocyte Lamellipod [Article]. Biophysical Journal. 2009 OCT 7;97(7):1853-1863.

[34] Larripa K, Mogilner A; Saha Inst Nucl Phys, Ctr Appl Math \& Computat Sci; Carl Zeiss India Pvt Ltd. Transport of a 1D viscoelastic actin-myosin strip of gel as a model of a crawling cell [Proceedings Paper]. Physica A-Statistical Mechanics and its Applications. 2006 DEC 1;372(1):113-123. Workshop on Common Trends in Traffic Systems, Kanpur, INDIA, FEB 08-10, 2006.

[35] Shao D, Rappel WJ, Levine H. Computational Model for Cell Morphodynamics [Article]. Physical Review Letters. 2010 SEP 2;105(10).

[36] Doubrovinski K, Kruse K. Cell Motility Resulting from Spontaneous Polymerization Waves. Phys Rev Lett. 2011 Dec;107:258103. Available from: http://link.aps.org/doi/10.1103/PhysRevLett.107.258103.

[37] Hawkins RJ, Poincloux R, Benichou O, Piel M, Chavrier P, Voituriez R. Spontaneous Contractility-Mediated Cortical Flow Generates Cell Migration in Three-Dimensional Environments. Biophysical Journal. 2011 SEP 7;101(5):1041-1045.

[38] Salbreux G, Joanny JF, Prost J, Pullarkat P. Shape oscillations of non-adhering fibroblast cells. Physical Biology. 2007;4(4):268. Available from: http://stacks.iop.org/1478-3975/4/i=4/a=004.

[39] Bois JS, Jülicher F, Grill SW. Pattern Formation in Active Fluids. Phys Rev Lett. 2011 Jan;106:028103. Available from: http: //link. aps.org/doi/10.1103/PhysRevLett.106.028103.

[40] George U, Stéphanou A, Madzvamuse A. Mathematical modelling and numerical simulations of actin dynamics in the eukaryotic cell. Journal of Mathematical Biology. 2013;66:547-593. Available from: http://dx.doi .org/10.1007/s00285-012-0521-1.

[41] Wolgemuth CW, Stajic J, Mogilner A. Redundant Mechanisms for Stable Cell Locomotion Revealed by Minimal Models. Biophysical Journal. 2011 AUG 3;101(3):545-553.

[42] Barnhart EL, Lee KC, Keren K, Mogilner A, Theriot JA. An Adhesion-Dependent Switch between Mechanisms That Determine Motile Cell Shape. PLoS Biol. 2011 05;9(5):e1001059. Available from: http://dx.doi.org/10.1371\%2Fjournal.pbio.1001059.

[43] Recho P, Truskinovsky L. Asymmetry between pushing and pulling for crawling cells. Phys Rev E. 2013 Feb;87:022720. Available from: http://link.aps.org/doi/10.1103/PhysRevE.87.022720.

[44] Recho P, Putelat T, Truskinovsky L. Motility initiation in active gels. submitted. 2015;

[45] Boal D. Mechanics of the Cell. Cambridge University Press; 2002. Available from: http: //www . amazon.com/exec/obidos/redirect? tag=citeulike07-20\&path=ASIN/0521796814

[46] Sheetz M, Sable J, Dobereiner H. Continuous membrane-cytoskeleton adhesion requires continuous accommodation to lipid and cytoskeleton dynamics. Annual Review of Biophysics and Biomolecular Structure. 2006;35:417-434.

[47] Prost J, Barbetta C, Joanny JF. Dynamical Control of the Shape and Size of Stereocilia and Microvilli. Biophysical Journal. 2007;93(4):1124 - 1133. Available from: http://www.sciencedirect.com/science/article/pii/S0006349507713711.

[48] Barnhart EL, Allen GM, Julicher F, Theriot JA. Bipedal Locomotion in Crawling Cells. Biophysical Journal. 2010 MAR 17;98(6):933-942.

[49] Du X, Doubrovinski K, Osterfield M. Self-Organized Cell Motility from Motor-Filament Interactions. Biophysical Journal. 2012;102(8):1738 - 1745. Available from: http://www.sciencedirect.com/science/article/pii/S0006349512003931.

[50] Loosley AJ, Tang JX. Stick-slip motion and elastic coupling in crawling cells. Physical Review E. 2012 SEP 10;86(3, Part 1).

[51] Jiang H, Sun SX. Cellular pressure and volume regulation and implications for cell mechanics. Biophysical journal. 2013;105(3):609-619.

[52] Stroka KM, Jiang H, Chen SH, Tong Z, Wirtz D, Sun SX, et al. Water permeation drives tumor cell migration in confined microenvironments. Cell. 2014;157(3):611-623.

[53] Thoresen T, Lenz M, Gardel M. Reconstitution of Contractile Actomyosin Bundles. Biophysical Journal. 2011;100(11):2698 - 2705. Available from: http://www.sciencedirect.com/science/article/pii/S0006349511004760.

[54] Mikhlin SG. Linear integral equations. Hindustan publishing corp.; 1960.

[55] Wang Y, Botvinick E, Zhao Y, Berns M, Usami S, Tsien R, et al. Visualizing the mechanical activation of Src. Nature. 2005 APR 21;434(7036):1040-1045.

[56] Bershadsky A, Kozlov M, Geiger B. Adhesion-mediated mechanosensitivity: a time to experiment, and a time to theorize. Current opinion 
in cell biology. 2006;18(5):472-481.

[57] Vicente-Manzanares M, Ma X, Adelstein RS, Horwitz AR. Non-muscle myosin II takes centre stage in cell adhesion and migration. Nature Reviews Molecular Cell Biology. 2009 NOV;10(11):778-790.

[58] Wolfenson H, Henis YI, Geiger B, Bershadsky AD. The Heel and Toe of the Cell's Foot: A Multifaceted Approach for Understanding the Structure and Dynamics of Focal Adhesions. Cell Motility and the Cytoskeleton. 2009 NOV;66(11):1017-1029.

[59] Shutova M, Yang C, Vasiliev JM, Svitkina T. Functions of Nonmuscle Myosin II in Assembly of the Cellular Contractile System. Plos One. 2012 JUL 13;7(7).

[60] Novak IL, Slepchenko BM, Mogilner A, Loew LM. Cooperativity between cell contractility and adhesion. Physical review letters. 2004;93(26):268109.

[61] Besser A, Schwarz US. Coupling biochemistry and mechanics in cell adhesion: a model for inhomogeneous stress fiber contraction. New Journal of Physics. 2007;9(11):425.

[62] Deshpande VS, Mrksich M, McMeeking RM, Evans AG. A bio-mechanical model for coupling cell contractility with focal adhesion formation [Article]. Journal of the Mechanics and Physics of Solids. 2008 APR;56(4):1484-1510.

[63] Risler T. Cytoskeleton and Cell Motility. arXiv:11052423. 2011;

[64] Verkhovsky A, Svitkina T, Borisy G. Self-polarization and directional motility of cytoplasm. Current Biology. 1999 JAN 14;9(1):11-20.

[65] Csucs G, Quirin K, Danuser G. Locomotion of fish epidermal keratocytes on spatially selective adhesion patterns. Cell Motility and the Cytoskeleton. 2007;64(11):856-867. Available from: http://dx.doi.org/10.1002/cm.20230.

[66] Lombardi ML, Knecht DA, Dembo M, Lee J. Traction force microscopy in Dictyostelium reveals distinct roles for myosin II motor and actin-crosslinking activity in polarized cell movement. Journal of cell science. 2007 May;120(Pt 9):1624-1634. Available from: http: //dx.doi.org/10.1242/jcs.002527.

[67] Yam PT, Wilson CA, Ji L, Hebert B, Barnhart EL, Dye NA, et al. Actin-myosin network reorganization breaks symmetry at the cell rear to spontaneously initiate polarized cell motility. Journal of Cell Biology. 2007 SEP 24;178(7):1207-1221.

[68] Jilkine A, Edelstein-Keshet L. A Comparison of Mathematical Models for Polarization of Single Eukaryotic Cells in Response to Guided Cues. PLoS Comput Biol. 2011 04;7(4):e1001121. Available from: http://dx.doi.org/10.1371\%2Fjournal.pcbi.1001121.

[69] Zhang W, Qian J, Yao H, Chen W, Gao H. Effects of functionally graded materials on dynamics of molecular bond clusters. SCIENCE CHINA Physics, Mechanics \& Astronomy. 2012;55:980-988. 10.1007/s11433-012-4726-5. Available from: http://dx.doi.org/10. $1007 / \mathrm{s} 11433-012-4726-5$

[70] Bottino D, Mogilner A, Roberts T, Stewart M, Oster G. How nematode sperm crawl. Journal of Cell Science. 2002 JAN 15;115(2):367-384.

[71] Zajac M, Dacanay B, Mohler WA, Wolgemuth CW. Depolymerization-driven flow in nematode spermatozoa relates crawling speed to size and shape. Biophysical Journal. 2008 MAY 15;94(10):3810-3823.

[72] Fournier MF, Sauser R, Ambrosi D, Meister JJ, Verkhovsky AB. Force transmission in migrating cells [Article]. Journal of Cell Biology. 2010 JAN 25;188(2):287-297.

[73] Peschetola V, Laurent VM, Duperray A, Michel R, Ambrosi D, Preziosi L, et al. Time-dependent traction force microscopy for cancer cells as a measure of invasiveness. Cytoskeleton. 2013;70(4):201-214.

[74] Bellairs R. Michael Abercrombie (1912-1979) [Biographical-Item]. International Journal of Developmental Biology. 2000;44(1, Sp. Iss. SI):23-28.

[75] Alberts B, Johnson A, Lewis J, Raff M, Roberts K, Walter P. Molecular biology of the cell. 4th ed. Garland Science Taylor \& Francis Group; 2002.

[76] Ambrosi D, Duperray A, Peschetola V, Verdier C. Traction patterns of tumor cells. Journal of mathematical biology. 2009;58(1-2):163-181. 